\title{
Púrpura trombocitopénica trombótica versus síndrome HELLP: reto diagnóstico en el embarazo*
}

\author{
(D) Leidy De Jesus-Henriques, ${ }^{1}$ (D) Katherine A. Rosales-Pereira, ${ }^{1}$ (D) Diana De Oliveira-Gomes, ${ }^{1}$ \\ (D) Rocío Iglesias-Fortes, ${ }^{1}$ (D) Erik L Dávila Alcalá. ${ }^{2}$
}

\begin{abstract}
RESUMEN
A nivel mundial la trombocitopenia se encuentra entre una de las primeras causas de alteraciones hematológicas en el embarazo, afectando hasta un 10\% de los mismos. A pesar de que todas las entidades clinicas con trombocitopenia en la gestación poseen un espectro clínico parecido, la distinción entre ellas es vital para establecer el tratamiento adecuado y así disminuir la morbimortalidad materno-fetal. La púrpura trombocitopénica trombótica es una enfermedad infrecuente, que, a pesar de no estar ligada al embarazo, no está exenta de presentarse en el mismo, en comparación al síndrome HELLP el cual es una complicación multisistémica que se presenta durante la gestación. A continuación, se presenta el caso de una gestante con anemia trombocitopénica microangiopática donde se evidencia la similitud clínica de ambas patologías, representando un reto diagnóstico.
\end{abstract}

Palabras clave: Trombocitopenia, Embarazo, Síndrome HELLP, Púrpura trombocitopénica trombótica, Anemia.

\section{SUMMARY}

Worldwide, thrombocytopenia is one of the first causes of hematological alterations in pregnancy, affecting up to $10 \%$ of them. Despite the fact that all clinical entities with thrombocytopenia in pregnancy have a similar clinical spectrum, the distinction between them is vital to establish the appropriate treatment and thus reduce maternal-fetal morbidity and mortality. Purpura thrombotic thrombocytopenic is an infrequent disease, which despite not being linked to pregnancy, is not exempt from occurring in it, compared to HELLP syndrome, which is a multisystemic complication that occurs during pregnancy. The following is the case of a pregnant woman with microangiopathic thrombocytopenic anemia where the clinical similarity of both pathologies is evidenced, representing a diagnostic challenge.

Keywords: Thrombocytopenia, Pregnancy, HELLP Syndrome, Purpura thrombotic thrombocytopenic, Anemia.

\section{INTRODUCCIÓN}

La púrpura trombocitopénica trombótica (PTT) es una enfermedad inusual de mortales consecuencias;

\footnotetext{
${ }^{1}$ Médico Cirujano. Escuela Luis Razetti. Universidad Central de Venezuela (UCV). ${ }^{2}$ Médico Internista, Hospital Universitario de Caracas - UCV. Médico Internista de la Unidad de Pacientes Críticos del Hospital de San Carlos Dr. Benicio Arzola Medina, Servicio de Salud Ñuble, SantiagoChile. Hospital de Clínicas Caracas.

* Trabajo presentado como póster en el XXIII Congreso de Medicina Interna de la Sociedad Venezolana de Medicina Interna.
}

Forma de citar este artículo: De Jesús-Henriques L, RosalesPereira K, De Oliveira-Gomes D, Iglesias-Forte R, Dávila EL. Púrpura trombocitopénica trombótica versus síndrome HELLP: reto diagnóstico en el embarazo. Rev Obstet Ginecol Venez. 2021; 81(3):292-296. https://doi org/10.51288/00810313 no es una enfermedad propia de la gestación, pero sí un factor de riesgo durante la misma. La trombocitopenia se presenta como una anemia hemolítica microangiopática, que, en conjunto con anemia hemolítica autoinmune, signos neurológicos (confusión, cefaleas, convulsiones), fiebre y afectación renal, caracterizan la clínica de la PTT. Estos síntomas clásicos solo aparecen en el $40 \%$ de toda la población afectada, no mejoran tras el parto, y su tratamiento está enfocado principalmente en la plasmaféresis (1-3).

Por su parte, el síndrome HELLP (del inglés, hemolysis, elevated liver enzymes y low platelet count), propio de la gestación, es así mismo, un tipo 
de anemia microangiopática trombótica que consiste en un cuadro caracterizado por enzimas hepáticas elevadas, trombocitopenia y anemia hemolítica, aunque la sintomatología neurológica también puede estar asociada. En comparación con la PTT, la clínica del HELLP mejora tras el parto, siendo este el principal tratamiento en última instancia $(2,4)$.

Ambas patologías pueden presentarse con síntomas y signos clínicos similares. He allí el reto diagnóstico y terapéutico, los cuales, si no se realizan a tiempo, pueden generar resultados fatales. Se presenta una gestante de 34 semanas en la cual se pone en evidencia el reto diagnóstico que constituyen estas dos entidades clínicas.

\section{CASO CLÍNICO}

Se trata de paciente femenino de 43 años, natural de China y procedente de Caracas, con embarazo de 34 semanas de gestación y con historia obstétrica de IIG IC, sin patologías previas conocidas. Al interrogatorio, negó alergia a medicamentos, así como también el consumo de drogas ilícitas y hábito tabáquico. Ingresó a la emergencia por presentar contracciones uterinas dolorosas y efectivas. Al examen físico de ingreso, presión arterial: 220/119 $\mathrm{mm} \mathrm{Hg}$, frecuencia cardiaca: 132 latidos por minuto (lpm), frecuencia respiratoria: 26 por minuto, saturación de O2: $98 \%$ aire ambiente. La paciente estaba afebril al tacto, hidratada, en regulares condiciones generales. La evaluación otorrinolaringológica estaba dentro de límites normales. El examen cardiovascular reveló un tórax normoconfigurado, ruidos respiratorios presentes en ambos hemitórax, sin agregados; ruidos cardiacos rítmicos y regulares, sin soplos. En el abdomen, los ruidos hidroaéreos estaban presentes, el abdomen era globoso a expensas del útero gestante, la frecuencia cardíaca fetal estaba audible en $150 \mathrm{lpm}$. En las extremidades se observó edema grado II/IV. A la evaluación neurológica, la paciente estaba orientada en tiempo, espacio y persona, con lenguaje coherente, fuerza muscular V/V generalizada, sensibilidad conservada, reflejos osteotendinosos II/IV, sin signos de focalización.

Los resultados de los exámenes paraclínicos fueron: plaquetas $56000 / \mathrm{L}$, lactatodeshidrogensa (LDH) 814 $\mathrm{UI} / \mathrm{L}$, creatinina $1,18 \mathrm{mg} / \mathrm{dL}$, aspartato transaminasa (AST) $15 \mathrm{U} / \mathrm{L}$, alanina transaminasa (ALT) $48 \mathrm{U} / \mathrm{L}$, gammaglutamil-transpeptidasa (GGT) $178 \mathrm{U} / \mathrm{L}$, fosfatasa alcalina $158 \mathrm{U} / \mathrm{L}$, ácido úrico $7,29 \mathrm{mg} / \mathrm{dL}$, proteinuria +1 , hemoglobinuria +2 , el frotis de sangre periférica mostró trombocitopenia, plaquetas grandes, agregados plaquetarios, esquistocitos + , macrocitosis + y policromasia + .

En vista del cuadro clínico, la paciente fue sometida a una cesárea segmentaria. Se le realizó monitoreo de presión arterial, cuyos valores mejoraron al realizarse la cesárea segmentaria. Las cifras hematológicas se estabilizaron sin plasmaféresis ni esteroides, egresando a las 48 horas, estable.

\section{DISCUSIÓN}

La trombocitopenia constituye la segunda causa de las alteraciones hematológicas encontradas durante el embarazo, superada por la anemia, y es un motivo de consulta común para el hematólogo. Afecta el $10 \%$ de todos los embarazos y representa un reto diagnóstico y terapéutico, ya que puede estar relacionado a condiciones preexistentes en mujeres en edad fértil, como la trombocitopenia inmune primaria (TIP), el lupus eritematoso sistémico (LES) o la PTT, o estar presente en trastornos intrínsecos del embarazo, como la trombocitopenia gestacional o el síndrome HELLP (5-8).

La PTT es una enfermedad infrecuente (6/1 000000 habitantes/año en EE.UU.), que no posee criterios diagnósticos establecidos. Su fisiopatología consiste en un cuadro de microangiopatía trombótica que se 


\section{PÚRPURA TROMBOCITOPÉNICA TROMBÓTICA VERSUS SÍNDROME HELLP: RETO DIAGNÓSTICO EN EL EMBARAZO}

caracteriza por un proceso de agregación intravascular causada por el déficit congénito o adquirido de la ADAMTS-13 (disintegrin-like and metalloprotease with thrombospondin type 1 motif 1318), una metaloproteasa que regula el tamaño del factor von Willebrand. El déficit de la misma no fragmenta adecuadamente el factor von Willebrand, provocando la formación de trombos intravasculares, obstrucción de la microcirculación y el cuadro clínico (9-11).

Los 6 pilares clásicos que orientan en el diagnóstico de esta enfermedad son: trombocitopenia, anemia hemolítica microangiopática, prueba de antiglobulina negativa, alteraciones neurológicas, fallo renal (niveles de creatinina $3 \mathrm{mg} / \mathrm{dL}$ ) y fiebre. Aunque la paciente que se presenta tenían anemia hemolítica microangiopática, la misma no es específica de la PTT, pudiendo asociarse en este caso a enfermedades características de la gestación, por ejemplo, el síndrome HELLP (9-11).

El síndrome HELLP es una complicación multisistémica del embarazo que se caracteriza por hemólisis, elevación de las enzimas hepáticas y trombocitopenia. El $70 \%$ de los casos se presentan durante el embarazo y el $30 \%$ durante las primeras 48 horas del puerperio. Por lo general, se inicia durante el tercer trimestre, afectando del $0,5 \%$ al $0,9 \%$ de todos los embarazos y hasta el $20 \%$ de los embarazos complicados con preeclampsia grave. Se observa un pico de incidencia entre la semana 2737 de gestación, solo el $10 \%$ se presenta antes de la semana 27. La importancia del diagnóstico radica en la elevada mortalidad materna (1\% - $24 \%)$ y fetal $(7 \%$ - $34 \%)$ asociada $(2,12,13)$. A pesar de que la clínica es variable, el síntoma más común es el dolor y la distensión abdominal, fundamentalmente en región epigástrica e hipocondrio derecho o región subesternal, concomitantes náuseas, vómitos, mal estado general, aumento de peso y edema. Síntomas menos comunes incluyen: cefalea, alteraciones visuales, ictericia y ascitis. La hipertensión (presión arterial > 140/90 mm Hg) y la proteinuria están presentes en aproximadamente el $85 \%$ de los casos. Solo un $20 \%$ de los casos de HELLP grave cursan con presión arterial normal $(2,13-15)$. Su diagnóstico se basa en la presencia, en una mujer embarazada, de las alteraciones analíticas que componen su nombre.

La anemia hemolítica microangiopática con presencia de esquistocitos (hematíes fragmentados) en frotis de sangre periférica es la principal manifestación del síndrome. Esta hemólisis se asocia a elevación de LDH (> $600 \mathrm{UI} / \mathrm{L}$ ) y disminución en los valores séricos de haptoglobina $(<1 \mathrm{~g} / \mathrm{L})$. Una característica obligatoria es la trombocitopenia $(<150000 / \mathrm{L})$ secundaria al consumo y adhesión plaquetaria en el endotelio lesionado. De hecho, cuando la paciente presente recuento plaquetario $<50000 / \mathrm{L}$ o el fibrinógeno se encuentre por debajo de $300 \mathrm{mg} / \mathrm{dL}$, debe considerarse que está en proceso de coagulación intravascular diseminada (CID), condición que empeora el pronóstico. La elevación de las enzimas hepáticas se relaciona con la hemólisis o con la necrosis hepática secundaria a microangiopatía. El $30 \%$ puede mostrar aumento de fosfatasa alcalina (FA) y gammaglutamiltranspeptidasa (GGT). Sin embargo, en la mayoría de las pacientes, la función hepática se mantiene normal dado que la necrosis es focalizada. Otros hallazgos son proteinuria $75 \%$, aumento de creatinina, aumento de bilirrubina mayor o igual a $1,2 \mathrm{mg} / \mathrm{dL}$, e hiperuricemia. Recientes estudios sugieren la utilidad de biomarcadores angiogénicos en el diagnóstico diferencial de la trombocitopenia en el embarazo (2, $13-15)$.

Existen diversas clasificaciones, entre las más destacadas están la clasificación de Mississippi (Cuadro 1), la cual lo clasifica en tres clases y la de Tennessee (Cuadro 2) que considera el síndrome HELLP como incompleto si cumple solo 1 o 2 criterios de la triada de laboratorio y como completo si existen los 3 (15-17). 
Cuadro 1. Clasificación de Mississippi (16)

\begin{tabular}{lccc}
\hline Clase & Plaquetas & AST o ALT & LDH \\
\hline HELLP clase I & $\leq 50000 / \mathrm{L}$ & $\geq 70 \mathrm{U} / \mathrm{L}$ & $\geq 600 \mathrm{UI} / \mathrm{L}$ \\
HELLP clase II & 50000 a $100000 / \mathrm{L}$ & $\geq 70 \mathrm{U} / \mathrm{L}$ & $\geq 600 \mathrm{UI} / \mathrm{L}$ \\
HELLP clase III & 100000 a $150000 / \mathrm{L}$ & $\geq 40 \mathrm{U} / \mathrm{L}$ & $\geq 600 \mathrm{UI} / \mathrm{L}$
\end{tabular}

Por todo lo antes expuesto, en vista de la clínica, los hallazgos de laboratorio y la resolución del cuadro clínico en esta, paciente luego de la realización de la cesárea segmentaria, se llegó al diagnóstico de síndrome HELLP, sin embargo, no se podría clasificar según Mississippi ya que no cumple con todos los criterios de una misma clase, pero según Tennessee se clasifica en síndrome HELLP incompleto, ya que los valores de AST no se encontraron elevados. Como se menciona anteriormente, el tratamiento de esta entidad clínica consiste en interrumpir la gestación, estabilizando previamente a la madre con: 1) tratamiento hipotensor, 2) tratamiento anticonvulsivante, 3) tratamiento con corticoides a altas dosis (betametasona $12 \mathrm{mg} / 12$ horas o dexametasona $10 \mathrm{mg} / 12$ horas, por 48 horas si es preciso para maduración pulmonar fetal, seguido de metilprednisolona $40 \mathrm{mg} / 12$ horas, si el tratamiento debe prolongarse). Es importante tomar en cuenta que nunca debe darse como curado un HELLP a pesar de que en ocasiones la analítica mejore con la medicación. Solo se cura al terminar la gestación (18).

Cuadro 2. Clasificación de Tennessee (17)

\begin{tabular}{ll}
\hline AST & $\geq 70 \mathrm{U} / \mathrm{L}$ \\
$\mathrm{LDH}$ & $\geq 600 \mathrm{UI} / \mathrm{L}$ \\
Plaquetas & $\leq 100000 / \mathrm{L}$ \\
\hline
\end{tabular}

\section{CONCLUSIÓN}

La púrpura trombocitopénica trombótica debe considerarse como diagnóstico diferencial en toda gestante con trombocitopenia, anemia gravey elevación de LDH junto a la mínima elevación de AST. Esta enfermedad es 3 veces más frecuente en mujeres en edad fértil, por esta razón esta patología no está exenta de presentarse en el embarazo, tanto en exámenes de rutina como en exacerbación de patologías durante el mismo. Las pacientes que han sufrido PTT tienen más riesgo de presentar recurrencia en posteriores gestaciones, aunque no se conoce exactamente el porcentaje del riesgo, la distinción entre esta entidad y la preeclampsia/HELLP es importante en cuanto a manejo y pronóstico, sin embargo, las características clínicas e histológicas son tan similares que a menudo establecer el diagnóstico correcto constituye un reto.

\section{REFERENCIAS}

1. Sadler JE, Moake JL, Miyata T, George JN. Recent advances in thrombotic thrombocytopenic purpura. Hematology Am Soc Hematol Educ Program. 2004:407-423. doi: 10.1182/asheducation-2004.1.407.

2. Belmonte L, Moreno R, Morón M, Callejón C, González G. Púrpura trombótica trombocitopénica versus síndrome de HELLP: un reto diagnóstico durante la gestación. Prog Obstet Ginecol. 2014; 57(4):180-184. dOI: 10.1016/j.pog.2013.11.007

3. Kelton JG. Idiopathic thrombocytopenic purpura complicating pregnancy. Blood Rev. 2002; 16(1):4346. doi: 10.1054/blre.2001.0181. 


\section{PÚRPURA TROMBOCITOPÉNICA TROMBÓTICA VERSUS SÍNDROME HELLP: RETO DIAGNÓSTICO EN EL EMBARAZO}

4. Sibai B, Dekker G, Kupferminc M. Pre-eclampsia. Lancet. 2005; 365(9461):785-799. doi: 10.1016/S01406736(05)17987-2.

5. Gernsheimer T, James AH, Stasi R. How I treat thrombocytopenia in pregnancy. Blood. 2013; 121(1):38-47. doi: 10.1182/blood-2012-08-448944.

6. Bockenstedt PL. Thrombocytopenia in pregnancy. Hematol Oncol Clin North Am. 2011; 25(2):293-310, vii-viii. doi: 10.1016/j.hoc.2011.01.004.

7. Myers B. Diagnosis and management of maternal thrombocytopenia in pregnancy. Br J Haematol. 2012; 158(1):3-15. doi: 10.1111/j.1365-2141.2012.09135.x.

8. Hott S, Queirolo A, Veas N, Rubio G. Púrpura trombocitopénico idiopático y embarazo. Cesárea versus parto vaginal. Rev Chil Obstet Ginecol. 2006; 71(5):327-332. http://dx.doi.org/10.4067/S071775262006000500006

9. Almagro D. Algunas consideraciones acerca de la púrpura trombocitopénica trombótica. Rev Cubana Hematol Inmunol Hemoter [Internet]. 2007 [4 de julio de 2020]; 23(1):22-30. Disponible en: $\quad$ http://scielo.sld.cu/scielo.php?script=sci arttext\&pid=S0864-02892007000100002

10. Moake JL. Thrombotic microangiopathies. N Engl J Med. 2002; 347(8):589-600. doi: 10.1056/ NEJMra020528.

11. de la Rubia J, Contreras E, Del Río-Garma J. [Thrombotic thrombocytopenic purpura]. Med Clin (Barc). 2011; 136(12):534-40. Spanish. doi: 10.1016/j. medcli.2010.02.011.

12. Abildgaard U, Heimdal K. Pathogenesis of the syndrome of hemolysis, elevated liver enzymes, and low platelet count (HELLP): a review. Eur J Obstet Gynecol Reprod Biol. 2013; 166(2):117-123. doi: 10.1016/j.ejogrb.2012.09.026.
13. Benedetto C, Marozio L, Tancredi A, Picardo E, Nardolillo P, Tavella AM, et al. Biochemistry of HELLP syndrome. Adv Clin Chem. 2011; 53:85-104. doi: 10.1016/b978-0-12-385855-9.00004-7.

14. Broughton Pipkin F, Roberts JM. Hypertension in pregnancy. J Hum Hypertens. 2000; 14(10-11):705724. doi: 10.1038/sj.jhh.1001018.

15. Sibai BM. Diagnosis, controversies, and management of the syndrome of hemolysis, elevated liver enzymes, and low platelet count. Obstet Gynecol. 2004; 103(5 Pt 1):981-991. doi: 10.1097/01. AOG.0000126245.35811.2a.

16. Martin JN Jr, Rinehart BK, May WL, Magann EF, Terrone DA, Blake PG. The spectrum of severe preeclampsia: comparative analysis by HELLP (hemolysis, elevated liver enzyme levels, and low platelet count) syndrome classification. Am J Obstet Gynecol. 1999; 180(6 Pt 1):1373-1384. doi: 10.1016/ s0002-9378(99)70022-0.

17. Sibai BM. The HELLP syndrome (hemolysis, elevated liver enzymes, and low platelets): much ado about nothing? Am J Obstet Gynecol. 1990; 162(2):311-316. doi: 10.1016/0002-9378(90)90376-i.

18. Cararach V, Botet F. Preeclampsia. Eclampsia y síndrome HELLP. En: Asociación Española de Pediatría, editores. Protocolos Diagnósticos Terapéuticos de la AEP: Neonatología. Barcelona: AEP; 2008 [consultado 11 de julio de 2020]. p 139-144. Disponible en: https:// www.aeped.es/sites/default/files/documentos/16_1.pdf

Recibido 7 de noviembre de 2020 Aprobado 10 de febrero de 2021 